\title{
Plantas medicinais como opção terapêutica em comunidade de Montes Claros, Minas Gerais, Brasil
}

PIRES, I.F.B.1; SOUZA, A.A.1; FEITOSA, M.H.A.2; COSTA, S.M. ${ }^{3}$

Universidade Estadual de Montes Claros, Unimontes, Centro de Ciências Biológicas e da Saúde, CCBS. Campus Universitário Professor Darcy Ribeiro. Vila Mauricéia. Montes Claros, Minas Gerais, Brasil. Caixa Postal 126CEP: 39401-089; 'Departamento de Fisiopatologia da Universidade Estadual de Montes Claros, Unimontes, mhafeitosa@ig.com.br Rua João F. Pimenta, n 378/201. Bairro Cidade Santa Maria, Montes Claros, Minas Gerais, Brasil, CEP: 39401081. Telefones: 38- 91340403, 38- 32228842; 'Programa de Pós-graduação em Cuidado Primário em Saúde, Departamento de Odontologia, Universidade Estadual de Montes Claros, Unimontes.

RESUMO: Este trabalho objetivou identificar a importância de plantas medicinais como primeira linha de ação para enfermidades em população residente no território da Estratégia Saúde da Família (ESF) de Montes Claros/MG, Brasil. A pesquisa foi transversal e descritiva, realizada em 2010, sendo a coleta de dados obtida por questionário semi-estruturado. O cálculo amostral do cenário do estudo embasou-se no número de famílias da ESF. O tratamento estatístico considerou o nível de significância $p<0,05$. Para cada família $(\mathrm{N}=253)$ foi entrevistada uma pessoa. Dos entrevistados, $75,5 \%$ utilizam plantas medicinais; sem associação com renda, cor de pele, escolaridade e sexo $(p>0,05)$. Quase a metade dos entrevistados $(45,1 \%)$ informou que utiliza mais de uma planta por vez. No caso de doença, após consulta e prescrição médica, uma parcela importante dos usuários de plantas (48,7 \%) usa apenas o medicamento alopático. Concluiu-se que a utilização de plantas medicinais como recurso terapêutico independe de escolaridade e renda familiar, sugerindo incorporação cultural da prática integrativa na comunidade nortemineira estudada.

Palavras-chave: fitoterapia, opção terapêutica, plantas medicinais.

ABSTRACT: Medicinal plants as a therapeutic option in the community Montes Claros, Minas Gerais, Brazil. This study aimed to identify the importance of medicinal plants as a first line of action when treating diseases in populations residing in the territory of the Family Health Strategy (FHS) in Montes Claros,/state of Minas Gerais, Brazil.tThis is a cross-sectional descriptive research. Data collection took place by means of a semi-structured questionnaire in 2010. The sample size calculation was based on the number of families in the FHS study setting. For statistical analysis, the significance level considered was $p<0.05$. One personofrom each family (253) was interviewed. Of the respondents, regardless of income, skin color, education and sex, $75.5 \%$ stated they use medicinal plants ( $p>0.05$ ). Almost half of respondents $(45.1$ $\%$ ) reported the use of more than one plant at a time. In the event of illness, $42.3 \%$ reported to use medicinal plants and visit the doctor. In case of illness, after consultation and prescription, a significant portion of the plant users $(48.7 \%)$ uses only allopathic medicine.sWe concluded that the use of medicinal plants as a therapeutic resource is independent of education and income, which suggestg cultural incorporation of the integrative practice in the community studied.

Keywords: phytotherapy, therapeutic option, medicinal plants.

\section{INTRODUÇÃO}

O uso de plantas medicinais na cura de enfermidades é uma forma antiga de tratamento fundamentada em informações provenientes de sucessivas gerações (Pinto et al., 2006; Tresvenzol et al.,2006; Ceolin et al. ,2011). Civilizações mais antigas já sabiam que algumas plantas continham princípios ativos com poder curativo no combate às doenças (Badke et al.,2011). Sendo assim, o uso de plantas medicinais remonta ao início da própria origem dos seres humanos (Dantas \& Guimarães, 2006) e constitui, muitas vezes, o único recurso terapêutico para determinadas comunidades (Lopez, 
2006).

A importância das plantas medicinais para estas populações tem sido revelada por estudos etnobotânicos realizados em diversas regiões brasileiras, abrangendo os biomas como: Mata Atlântica (Costa \& Mayworm, 2011), Cerrado (Cunha \& Bortolotto, 2011), Caatinga (Marinho et al., 2011), Pantanal (Oliveira et al., 2011) e Amazônia (CoelhoFerreira, 2009; Costa \& Mitja, 2010; Evangelista et al., 2013).

As plantas medicinais, como recurso terapêutico, despertam o interesse para as investigações no intuito de desenvolver fitoterápicos (Bittencourt et al.,2002); de documentar as plantas, compreender como elas são percebidas nas comunidades, identificar a importância delas no contexto da medicina tradicional e de sua incorporação no setor de saúde pública, principalmente na Atenção Primária à Saúde no âmbito do Sistema Único de Saúde (SUS).

Ao reconhecer a importância do uso de plantas medicinais junto à população brasileira, o governo federal legalizou a fitoterapia no Sistema Único de Saúde (SUS), em 2005. A Política Nacional de Medicina Natural e Práticas Complementares no SUS foi renomeada, posteriormente, como Política Nacional de Plantas Medicinais e Fitoterápicos (PNPMF) (Brasil 2005; 2006). A PNPMF foi avaliada pela Agência Nacional de Vigilância Sanitária (ANVISA), que normatizou registros de medicamentos fitoterápicos. As normas da ANVISA foram avaliadas pelo grupo de Carvalho et al., (2011), que atualizou os requisitos para comprovar a segurança e eficácia de medicamentos.

A incorporação da prática integrativa da fitoterapia no SUS culminou na necessidade de pesquisas nessa área com o intuito de enriquecer o conhecimento dos profissionais da saúde, além de tornar as práticas fitoterápicas mais seguras e eficazes (Calixto, 2000; Santos et al., 2011). O objetivo deste trabalho foi identificar o uso de plantas medicinais como primeira linha de ação às enfermidades em um território da Estratégia Saúde da Família de Montes Claros, Minas Gerais, Brasil.

\section{MATERIAL E MÉTODOS}

O protocolo desta pesquisa foi aprovado pelo Comitê de Ética em Pesquisa da Universidade Estadual de Montes Claros - Unimontes, parecer no 1189/2008, de acordo com a Resolução 196/96 do Conselho Nacional de Saúde (Brasil, 1996) e a Declaração de Helsink. O Termo de Consentimento Livre e Esclarecido foi obtido, individualmente, conforme proposto na Resolução 196/96, que normaliza a pesquisa em seres humanos.

Trata-se de estudo etnodirigido realizado a partir da pesquisa com abordagem quantitativa, de caráter observacional e transversal. A pesquisa constou de um levantamento de dados sobre o uso de plantas medicinais como primeira linha de ação às enfermidades no Bairro Cintra, em Montes Claros/MG, Brasil. A cidade de Montes Claros está situada na região Norte de Minas Gerais, sendo considerada a cidade pólo da área mineira da Agência de Desenvolvimento do Nordeste (ADENE ex-Superintendência de Desenvolvimento do Nordeste - SUDENE). Trata-se da quinta maior cidade do Estado e está situada a $426 \mathrm{~km}$ da capital Belo Horizonte, contando com um contingente populacional de 355.401 habitantes (IBGE, 2010). A vegetação que predomina no município é um mosaico de cerrado e caatinga.

A escolha do Bairro Cintra como objeto de estudo deu-se em decorrência do trabalho de territorialização feito pelo acadêmico de medicina, autor deste estudo, facilitando dessa forma a coleta de dados em domicílios, já que o local, cenário do estudo, é espaço de integração ensino e serviço. Quanto à situação sanitária, o bairro apresenta rede de esgoto, coleta de lixo e água tratada. Conta também com o serviço de saúde pública, no nível da atenção primária, por meio da Estratégia Saúde da Familia.

Para o cálculo do tamanho da amostra foi utilizado o Programa Estatístico Epi-info versão 6.0-DOS. O cálculo da amostra aleatória simples definiu 253 domicílios, representando 18,16\% de 1393 domicílios cadastrados na Estratégia Saúde da Família (ESF) do local estudado. Para o cálculo amostral, considerou-se uma prevalência de $50 \%$, estimativa de erro igual a $5 \%$, e intervalo de confiança igual a $95 \%$. Após definida a amostra, verificou-se o número de casas existentes em cada rua da área coberta pela ESF, e foi feita a alocação proporcional de domicílios a serem visitados em cada rua. A seleção das casas em cada rua seguiu o critério da amostra aleatória simples, por meio de sorteio pela numeração das casas. Cada uma destas representou uma família a ser entrevistada.

A partir da seleção dos domicílios, uma pessoa em cada casa foi entrevistada, considerando que as famílias têm hábitos comuns. Procurouse entrevistar prioritariamente a pessoa do sexo feminino responsável pela família; na sua ausência, porém, a segunda opção era o marido/companheiro e, como terceira opção, um(a) filho(a) maior de 18 anos. A prioridade pelas mulheres deu-se por elas serem consideradas as principais responsáveis pelo tratamento caseiro com uso de plantas medicinais (Pereira et al., 2005). Em adição, o conhecimento sobre as plantas medicinais está mais ligado às mulheres, que trocam mudas e receitas de remédios caseiros (Visbisk et al., 2003).O domínio

Rev. Bras. PI. Med., Campinas, v.16, n.2, supl. I, p.426-433, 2014. 
das mulheres nos primeiros cuidados foi comentado também por Coelho-Ferreira (2009).

Para a coleta de dados, aplicou-se questionário semi-estruturado sob a forma de entrevistas feitas em visita domiciliar pelos próprios pesquisadores treinados. Com o objetivo de avaliar a compreensão das perguntas, instruções e esclarecimentos necessários durante a aplicação do questionário, um estudo piloto foi realizado com dez indivíduos, que responderam ao questionário permitindo mudanças relacionadas à formulação de questões e alternativa de respostas, antes do estudo principal. A coleta de dados foi realizada no ano de 2010. O item "cor da pele" foi estimado conforme a percepção do próprio entrevistado. Variáveis independentes foram dicotomizadas como cor de pele (branca e não branca), escolaridade (até 11 anos de estudo e 12 ou mais anos), renda familiar mensal (até três salários mínimos- SM e quatro ou mais SM) e naturalidade (Montes Claros e outros municípios).

O tratamento estatístico foi realizado no Software Estatístico SPSS $₫$, versão 18.0, para Windows ${ }^{\circledR}$. Para a análise descritiva foram utilizadas as medidas de tendência central e de dispersão: média aritmética, desvio padrão, valores mínimos e máximos e quartis, bem como o cálculo de proporções. Para a associação entre as variáveis categóricas foram adotados os testes Qui-quadrado de Pearson, Regressão de Poisson com variância robusta, univariada e multivariada, e o teste de Wald. As razões de prevalência (RP) foram estimadas, assim como os respectivos intervalos de confiança a 95 \% (IC95 \%). Já, para a comparação de médias foi utilizado o teste $t$ Student (Pestana \& Gageiro, 2003). Para todos os testes adotou-se o nível de significância de $p<0,05$ (Hosmer \& Lemeshow, 2000).

\section{RESULTADOS}

Foram entrevistadas 253 pessoas correspondentes ao número de domicílios que compôs a amostra deste estudo. Todos os domicílios eram cadastrados na Estratégia Saúde da Família. A maioria era do sexo feminino, e de cor de pele "não branca" (parda, amarela, indígena, preta). (Tabela 1). A idade dos entrevistados variou de 18 a 89 anos, sendo a média igual a $49,32( \pm 18,205)$, a moda foi 62 anos, e os percentis $25 \%, 75 \%$ e $90 \%$ foram 33, 64 e 73 anos, respectivamente.

No que diz respeito à média de idade, conforme o sexo do entrevistado verificou-se que as mulheres tinham média de idade igual a $49,97( \pm 17,828)$ e os homens $45,66( \pm 20,045)$ sem diferença estatisticamente significativa entre os sexos $(p=0,179)$. Quanto à escolaridade dos entrevistados verificou-se que, a maior parcela possuía o primeiro grau incompleto $(41,1 \%)$, seguida do segundo grau incompleto $(24,5 \%)$, do primeiro grau completo $(11,1 \%)$, do terceiro grau incompleto $(7,5 \%)$, do segundo grau completo $(6,3 \%)$, do terceiro grau completo $(4,0 \%)$ e o analfabetismo foi constatado em $5,5 \%$ dos entrevistados. Desse modo, a maioria dos entrevistados $(64,0 \%)$ apresentou escolaridade equivalente até 11 anos (segundo grau incompleto). A renda mensal familiar relatada pela maioria $(75,5$ $\%$ ) dos entrevistados foi entre um a três salários mínimos.

Um pouco mais da metade dos entrevistados não é natural de Montes Claros (54,5\%) e relatou ter como principal atividade laboral "do lar" (Tabela 1).

Dos entrevistados, 75,5\% ( $\mathrm{N}=191)$ utilizam plantas medicinais (Figura 1). Quando avaliada a média de idade do entrevistado conforme o uso de plantas medicinais, verificou-se que, quem usa planta tem média de idade igual a 50,58 $( \pm 18,484)$ e os que não usam as plantas medicinais tem a média

TABELA 1. Distribuição dos entrevistados conforme o perfil. Indivíduos cadastrados em Estratégia Saúde da Família de Montes Claros, MG, Brasil, 2010.

\begin{tabular}{lll}
\hline Variáveis & Freqüência absoluta & Freqüência relativa \\
\hline Sexo & $\mathrm{n}$ & $\%$ \\
\hline Feminino & 214 & 84,6 \\
Masculino & 39 & 15,4 \\
\hline Cor de pele & $\mathrm{n}$ & $\%$ \\
\hline Branca & 119 & 47,0 \\
Não Branca & 134 & 53,0 \\
\hline Atividade laboral & $\mathrm{n}$ & $\%$ \\
\hline Do Lar & 124 & 49,0 \\
Outros & 129 & 51,0 \\
\hline Naturalidade & $\mathrm{n}$ & $\%$ \\
\hline Montes Claros & 115 & 45,5 \\
Outros & 138 & 54,5 \\
\hline
\end{tabular}

Rev. Bras. PI. Med., Campinas, v.16, n.2, supl. I, p.426-433, 2014. 
de idade igual $45,56( \pm 16,938)(p=0,058)$.

$\mathrm{Na}$ associação entre o uso de planta medicinal e as características do perfil dos entrevistados (Tabela 2), constatou-se que este uso foi associado à naturalidade do entrevistado, sendo que as pessoas que nasceram em Montes Claros apresentaram uma razão de prevalência 1,74 vezes

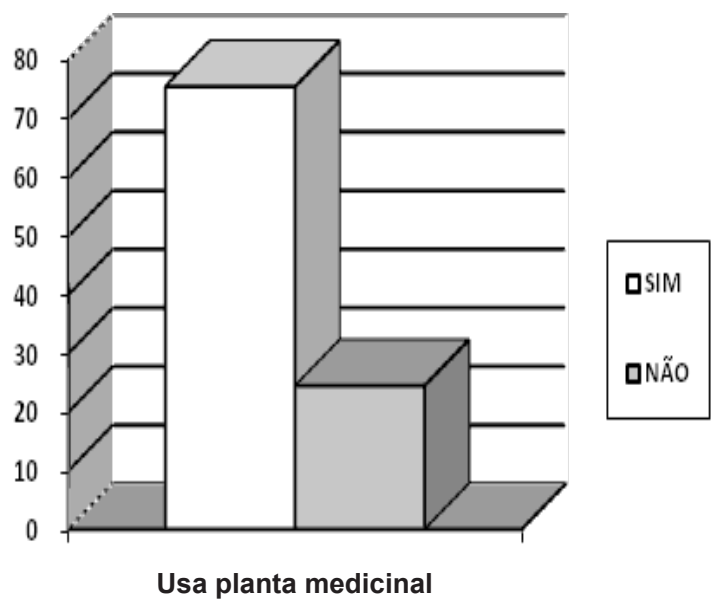

FIGURA 1. Distribuição dos pesquisados, em valores percentuais, conforme utilização de plantas medicinais. Indivíduos cadastrados em Estratégia Saúde da Família de Montes Claros, MG, Brasil, 2010. àquela apresentada por entrevistados procedentes de outros municípios.

Neste estudo, o uso de plantas medicinais não foi associado à renda mensal familiar, pois não foi encontrada associação estatisticamente significativa $(p=0,914)$ entre as variáveis (Tabela 3$)$.

Uma grande parcela $(45,1 \%)$ informou que utiliza mais de uma planta medicinal por vez. O uso de plantas medicinais também em condição de saúde, ou seja, na ausência de alguma doença específica, é uma prática utilizada por $46,2 \%$ dos entrevistados.

Entre os que utilizam as plantas medicinais (191), a principal forma de uso foi em chá $(94,8$ $\%$ ), contudo a garrafada foi também mencionada por 3,6 \%, sendo que 1,6\% não descreveram a principal forma de uso das plantas. A maioria dos entrevistados informou que o uso destas, melhora os sinais e sintomas das doenças $(73,1 \%)$ relacionadas aos sistemas do corpo humano, sendo o sistema respiratório o mais destacado $(45,8 \%)$, seguido pelo digestivo (12,6\%), cardiovascular $(6,8 \%)$ e excretor (2,1\%), também o psicológico foi considerado por $4,1 \%$ e $1,7 \%$ não responderam a essa questão. $\mathrm{O}$ destaque foi feito ao resfriado para aqueles que consideraram melhoras dos problemas no sistema respiratório.

Entre os 191 indivíduos que utilizam plantas

TABELA 2. Uso de plantas medicinais conforme o perfil do entrevistado. Regressão de Poisson univariada e multivariada com variância robusta. Indivíduos cadastrados em Estratégia Saúde da Família de Montes Claros, MG, Brasil, 2010.

\begin{tabular}{|c|c|c|c|c|}
\hline \multirow[t]{2}{*}{ Variáveis } & \multicolumn{2}{|c|}{ Usa plantas medicinais } & \multirow[t]{2}{*}{$\mathrm{RP}(95 \% \mathrm{IC})$ anivariada } & \multirow{2}{*}{$\begin{array}{l}\operatorname{RP}(95 \% \\
\text { multivariada }\end{array}$} \\
\hline & $\operatorname{Sim}(\%)$ & Não (\%) & & \\
\hline \multicolumn{5}{|l|}{$\overline{\text { Sexo }}$} \\
\hline Masculino & 66,7 & 33,3 & 1 & 1 \\
\hline \multirow[t]{2}{*}{ Feminino } & 76,5 & 23,5 & $0,70(0,42-1,17)$ & $0,69(0,41-1,14)$ \\
\hline & & & $p=0,174 b$ & $p=0,142^{b}$ \\
\hline \multicolumn{5}{|l|}{ Naturalidade } \\
\hline Outras cidades & 67,9 & 32,1 & 1 & 1 \\
\hline \multirow[t]{2}{*}{ Montes Claros } & 81,2 & 18,8 & $1,72(1,11-2,66)$ & $1,74(1,13-2,69)$ \\
\hline & & & $p=0.014^{b}$ & $p=0,012^{b}$ \\
\hline \multicolumn{5}{|l|}{ Escolaridade } \\
\hline$\geq 12$ anos & 75,8 & 24,2 & 1 & - \\
\hline \multirow[t]{2}{*}{$\leq 11$ anos } & 74,4 & 25,6 & $1.06(0,68-1,66)$ & - \\
\hline & & & $p=0,800^{b}$ & \\
\hline \multicolumn{5}{|l|}{ Cor de pele } \\
\hline Não branca & 76,1 & 23,9 & 1 & - \\
\hline \multirow[t]{2}{*}{ Branca } & 73,9 & 26,1 & $1,09(0,71-1,67)$ & - \\
\hline & & & $p=0,690^{b}$ & - \\
\hline
\end{tabular}

a RP (95 \% IC) - Razão de Prevalência (95 \% intervalo de confiança)

b Teste Wald, * Presença de perdas 
TABELA 3. Associação entre renda familiar mensal em salário mínimo (SM) e uso de plantas medicinais. Indivíduos cadastrados em Estratégia Saúde da Família de Montes Claros, MG, Brasil, 2010.

\begin{tabular}{llll}
\hline & \multicolumn{2}{l}{ Renda familiar mensal } & Teste Qui quadrado \\
\hline Usa plantas medicinais & $\leq 3 \mathrm{SM}$ & $\geq 4 \mathrm{SM}$ & $\mathrm{p}$ \\
& $\mathrm{n}(\%)$ & $\mathrm{n}(\%)$ & \\
\hline Sim & $164(86,8)$ & $25(13,2)$ & 0,914 \\
Não & $55(87,3)$ & $8(12,7)$ & \\
\hline Total & $\mathbf{2 1 9 ( 8 6 , 9 )}$ & $\mathbf{3 3 ( 1 3 , 1 )}$ & \\
\hline
\end{tabular}

medicinais, 48,6 \% afirmaram que ao procurar por assistência médica informam aos profissionais de saúde a utilização destas. Após a prescrição médica, uma parcela importante $(48,7 \%)$ dos usuários desta categoria de plantas passa a usar somente o medicamento prescrito pelo profissional de saúde; no entanto, 45,0 \% utilizam-nas além do medicamento prescrito e apenas $6,3 \%$ continuam a usar somente a planta medicinal.

O uso de medicamentos ou de plantas medicinais após a prescrição médica foi associado ao perfil do entrevistado (Tabela 4) e foi constatado que a postura do entrevistado quanto ao tipo de terapia a seguir, após uma prescrição medicamentosa, independe da cor de pele, sexo, renda e escolaridade $(p>0,05)$.

\section{DISCUSSÃO}

A maioria dos entrevistados é do sexo feminino, o que era esperado uma vez que a metodologia privilegiou a entrevista com a dona da casa. Esse critério foi adotado com embasamento na literatura, que informa ser a mulher a figura responsável pelo cultivo e preparo das plantas medicinais, assim como pela alimentação e cuidados dispensados às crianças e outros familiares, quando enfermos (Dias, 1999). Desse modo, apesar da entrevista ter sido feita com apenas um membro da família, os resultados apresentados podem ser inferidos para a perspectiva familiar.

Além dos adultos, a pesquisa contou com a participação de idosos (acima de 60 anos), o que pode ser explicado pelo fato da pesquisa ter sido

TABELA 4. Associação entre variáveis do perfil do entrevistado e conduta terapêutica após prescrição medicamentosa alopática. Indivíduos cadastrados em Estratégia Saúde da Família de Montes Claros, MG, Brasil, 2010.

\begin{tabular}{|c|c|c|c|c|}
\hline & \multicolumn{3}{|c|}{ Conduta terapêutica após prescrição medicamentosa alopática } & Teste Qui Quadrado \\
\hline Perfil do & Usa só remédio & Usa remédio e plantas & Usa só plantas & \multirow[b]{3}{*}{$\mathrm{p}$} \\
\hline entrevistado & $\mathrm{n}(\%)$ & $\mathrm{n}(\%)$ & $\mathrm{n}(\%)$ & \\
\hline & & & & \\
\hline \multicolumn{5}{|l|}{ Sexo } \\
\hline Feminino & $82(50,0)$ & $73(44,5)$ & $9(5,5)$ & \\
\hline Masculino & $11(40,7)$ & $13(48,1)$ & $3(11,1)$ & 0,440 \\
\hline Total & $93(48,7)$ & $86(45,0)$ & $12(6,3)$ & \\
\hline \multicolumn{5}{|l|}{ Cor } \\
\hline Branca & $44(50,6)$ & $37(42,5)$ & $6(6,9)$ & \\
\hline Não branca & $49(47,1)$ & $49(47,1)$ & $6(6,8)$ & 0,805 \\
\hline Total & $93(48,7)$ & $86(45,0)$ & $12(6,3)$ & \\
\hline \multicolumn{5}{|c|}{ Renda mensal } \\
\hline$\leq 3 \mathrm{SM}$ & $80(48,2)$ & $74(44,6)$ & $12(7,2)$ & \\
\hline$\geq 4 \mathrm{SM}$ & $13(54,2)$ & $11(45,8)$ & $0(0,0)$ & 0,388 \\
\hline Total & $93(48,9)$ & $85(44,8)$ & $12(6,3)$ & \\
\hline \multicolumn{5}{|l|}{ Escolaridade } \\
\hline$<12$ anos & $61(50,8)$ & $53(44,2)$ & $6(5,0)$ & \\
\hline$\geq 12$ anos & $31(44,9)$ & $32(46,4)$ & $6(8,7)$ & 0,521 \\
\hline Total & $92(48,7)$ & $85(45,0)$ & $12(6,3)$ & \\
\hline
\end{tabular}

Rev. Bras. PI. Med., Campinas, v.16, n.2, supl. I, p.426-433, 2014. 
conduzida no âmbito domiciliar, o que aumenta a chance de encontrar a população idosa, por estar em inatividade laboral.

Quanto à escolaridade dos entrevistados, o terceiro grau completo e a falta de escolaridade (analfabetismo) foram encontrados na menor parcela dos entrevistados, sendo o primeiro grau incompleto a escolaridade mais freqüente. Desse modo, pode-se afirmar que o estudo foi conduzido em região com pessoas com baixa escolaridade, assim como de baixa renda mensal familiar, entre um a três salários mínimos (SM) vigentes na época do estudo ( $S M=R \$ 510,00)$. A principal atividade laboral foi a dedicação às atividades do lar, que vem ao encontro do perfil socioeconômico dos entrevistados, baixa escolaridade e baixa renda mensal. A condição do entrevistado de apresentar uma renda familiar mensal acima de três salários mínimos não foi associada ao uso ou não de plantas medicinais, o que significa que o uso de planta independe da renda familiar mensal.

O uso de plantas medicinais foi detectado para grande maioria dos entrevistados e foi associado somente à condição da procedência do sujeito entrevistado, sendo que as pessoas que afirmaram ter nascido em Montes Claros apresentaram uma maior prevalência de respostas positivas para o uso de plantas. Essa associação pode ser explicada, em parte, por Montes Claros ser uma cidade caracterizada pelo forte histórico cultural, enraizado na sua população. Quanto à tradição de uso das plantas medicinais, o mercado central do município manifesta este aspecto, por meio das bancas de raízeiros existentes, que comercializam diferentes tipos de plantas cultivadas na região do Norte de Minas Gerais, na forma de sementes, raizes, cascas e folhas.

No Brasil, considerando a ampla diversidade de espécies vegetais, bem como a riqueza étnica e cultural, as plantas medicinais devem ocupar posição de destaque em relação à importância do uso popular medicinal (Garlet \& Irgang, 2001)

O uso de mais de uma planta medicinal, por vez, foi relatado por quase metade dos entrevistados, que utilizam também as plantas quando não estão doentes. Como exemplos deste fato, podem ser mencionados o uso do chá de hortelã e de garrafadas de aguardente com jurubeba.

Quanto à indicação terapêutica, quando uma planta medicinal apresenta um índice de concordância relativamente alto entre os informantes, sugere-se a real efetividade da planta no tratamento da doença (Friedman et al.,1986).

Neste estudo, foi verificado que os indivíduos fazem uso de plantas medicinais para o tratamento de diversos sistemas do corpo humano, sendo o sistema respiratório o mais destacado.
O chá (cozimento em água) foi a forma de utilização de plantas medicinais mais relatada na comunidade estudada. Essa forma de preparação das plantas vai ao encontro do estudo de Visbiski et al. (2003), que também identificaram ser os preparados crus ou frios, cozidos ou quentes a partir do cozimento em água a forma mais utilizada. No entanto, em outros estudos, outras formas de uso foram identificadas como as mais comuns, o xarope caseiro (Marinho et al., 2011) e a infusão (Costa \& Mayworn, 2011).

Apesar da maior parcela abandonar o uso das plantas medicinais após a prescrição médica alopática, deve-se destacar um resultado importante: a renda familiar mensal não influenciou na decisão quanto à continuidade do uso de planta medicinal. Isto demonstra que a escolha do uso das plantas medicinais, como linha terapêutica, perpassa mais pela questão cultural que pela questão financeira do indivíduo. Tal procedimento foi também observado no estudo de Visbiski et al. (2003), em que uma pessoa atingida por uma doença busca primeiramente a cura por meio dos remédios caseiros e, como segunda opção, procura por um profissional de saúde. Contudo, este agente acaba por priorizar a prescrição de medicamentos alopáticos, uma vez que o modelo hegemônico de formação não enfatiza as práticas integrativas e complementares na assistência à saúde.

Um pouco menos da metade dos entrevistados afirmou que ao procurar a assistência médica informa aos profissionais de saúde o uso de plantas medicinais. Essa informação é importante porque de certo modo pode despertar nos profissionais o interesse para o conhecimento de terapias alternativas e complementares, como a fitoterapia, que tem recebido destaque nas políticas públicas de saúde.

Com a Política Nacional de Práticas Integrativas e Complementares no SUS espera-se que os profissionais que atuam junto ao Sistema Único de Saúde (SUS) sejam capacitados para o uso adequado das plantas medicinais (Brasil, 2005). Cabe aos profissionais orientar as pessoas quanto ao uso indiscriminado de plantas e esclarecer as dúvidas da população quanto ao uso correto (Arnous et al., 2005). Para isso, faz-se necessária uma reformulação da estrutura curricular da atual formação em saúde, que no geral negligencia os conteúdos programáticos relacionados às práticas alternativas em saúde.

O presente estudo apresenta limitações por tratar-se de desenho transversal, de forma que, os resultados aqui apresentados não podem ser tomados como causa e efeito. Há possibilidade de viés de informação, uma vez que os dados trabalhados foram obtidos a partir de aplicação de

Rev. Bras. PI. Med., Campinas, v.16, n.2, supl. I, p.426-433, 2014. 
questionário junto à comunidade. Para controlar esse tipo de viés, houve um treinamento prévio, estudo piloto, entre os pesquisadores, para padronizar a conduta de abordagem ao participante da pesquisa. A pesquisa foi realizada em apenas um bairro de Montes Claros, limitando a generalização dos resultados para o município, contudo, o estudo tem validade interna, uma vez que se adotou o cálculo amostral para definir o tamanho de uma amostra que fosse representativa da comunidade, com seleção dos participantes pelo método aleatório simples.

O estudo traz contribuições importantes ao identificar o grande percentual de pessoas que utiliza plantas medicinais como recurso terapêutico, independente da escolaridade e renda familiar. Esse achado sugere que, houve incorporação cultural da prática integrativa na comunidade nortemineira. Ademais, reforça a importância da implantação da Política de Prática Integrativa e Complementar no âmbito do SUS e necessidade de educação permanente para o profissional trabalhador da rede pública de saúde.

\section{REFERÊNCIA}

ARNOUS, A.H. et al. Plantas medicinais de uso caseiro - conhecimento popular e interesse por cultivo comunitário. Revista Espaço para a Saúde, v.6, n.2, p.1-6, 2005.

BADKE, M.R. et al. Plantas medicinais: o saber sustentado na prática do cotidiano popular. Escola Anna Nery, v.15, n.1, p.132-9, 2011.

BITTENCOURT, S.C.et al. O uso das plantas medicinais sob prescrição médica: pontos de diálogo e controvérsias com uso popular. Revista Brasileira de Farmacognosia, v.12, supl., p.89-91, 2002.

BRASIL. Conselho Nacional de Saúde. Ministério da Saúde. Resolução n 196/96. Sobre pesquisa envolvendo seres humanos. Brasília. 1996, 24p.

BRASIL. Ministério da Saúde. Proposta de Política de Medicina Natural e Práticas Complementares no SUS, Brasília: Ministério da Saúde, 2005, 49p.

BRASIL. 2006. Diário Oficial da União. Decreto 5813, de 22 de junho de 2006. Disponível em: http://elegis.anvisa. gov.br/leisref/public/showAct.php?id=22681\&word.

Acesso em: 30 jan. 2007.

CALIXTO, J.B. Efficacy, safety, quality control, marketing and regulatory guidelines for herbal medicines (phytotherapeutic agents). Brazilian Journal of Medical and Biological Research, v.33, n.2, p.17989, 2000.

CARVALHO, A.C.B. et al. Regulation of herbal medicines in Brazil: advances and perspectives. Brazilian Journal of Pharmaceutical Sciences, v.47, n.3, p.467-73, 2011. Disponível em: http://www. scielo.br/scielo.php?script=sci_arttext\&pid=S1984$82502011000300004 \&$ Ing=en. Acesso em: 22 maio 2012.
CEOLIN, T. et al. Plantas Medicinais: transmissão do conhecimento nas famílias de agricultores de base ecológica no Sul do RS. Revista da Escola de Enfermagem da USP, v.45, n.1, p.47-54, 2011.

COELHO-FERREIRA, M. Medicinal knowledge and plant utilization in an Amazonian coastal community of Maruda, Para State (Brazil), Journal of Ethnopharmacology, v. 126, p.159-175, 2009.

COSTA, V.P.; MAYWORM, M.A.S. Plantas medicinais utilizadas pela comunidade do bairro dos Tenentes - município de Extrema, MG, Brasil. Revista Brasileira de Plantas Medicinais, v.13, n.3, p.282-92, 2011. Disponível em: http://www. scielo.br/scielo.php?script=sci_arttext\&pid=S1516$05722011000300006 \&$ Ing=en. Acesso em: 20 abr. 2012.

COSTA, J.R.; MITJA, D. Uso dos recursos vegetais por agricultores familiares de Manacapuru (AM). Acta Amaz., Manaus, v. 40, n. 1, p. 49-58,. 2010. Disponível em: http://www.scielo.br/scielo.php?script=sci arttext\&pid=S0044-59672010000100007\&Ing=en\& $\overline{n r}$ m=iso. Acesso em: 06 dec. 2013.

CUNHA, S.A.; BORTOLOTTO, I.M. Etnobotânica de Plantas Medicinais no Assentamento Monjolinho, município de Anastácio, Mato Grosso do Sul, Brasil. Acta Botanica Brasilica, v.25, n.3, p.685-98, 2011. Disponível em: http://www.scielo.br/scielo.php?script=sci_ arttext\&pid=S0102-33062011000300022\&Ing=en\& $\overline{n r}$ m=iso. Acesso em: 01 mai. 2012.

DANTAS, I.C.; GUIMARÃES, F.R. Perfil dos raizeiros que comercializam plantas medicinais no município de Campina Grande, PB. Revista de Biologia e Ciências da Terra, v.6, n.1, p.39-44, 2006.

DIAS, M.C. Plantas medicinais utilizadas no distrito de Juquiratiba - Município de Conhas - SP. 1999, 82 p. Mestrado em Agronomia - Dissertação - Universidade Estadual Paulista, Botucatu.

EVANGELISTA, S. S. et al . Fitoterápicos na odontologia: estudo etnobotânico na cidade de Manaus. Rev. bras. plantas med., Botucatu , v.15, n..4, p.513-9, 2013 . Disponível em http://www. scielo.br/scielo.php?script=sci_arttext\&pid=S151605722013000400007\&lng=pt\&nrm=iso. Acesso em : 06 dez. 2013.

FRIEDMAN, J. et al. A preliminary classification of the healing potential of medicinal plants, based on a rational analysis of an ethnopharmacological field survey among bedouins in the negev desert, Israel. Journal of Ethnopharmacology, v.16, n.1, p.275-87, 1986.

GARLET, T.M.B.; IRGANG, B.E. Plantas medicinais utilizadas na medicina popular por mulheres trabalhadoras rurais de Cruz Alta, Rio Grande do Sul, Brasil. Revista Brasileira de Plantas Medicinais, v.4, n.1, p.9-18, 2001.

HOSMER, D.W.; LEMESHOW, S. Applied Logistic Regression. 2.ed. New York: Wiley-Interscience Publication, 2000, 280p.

INSTITUTO BRASILEIRO DE GEOGRAFIA E ESTATÍSTICA. Censo populacional do Brasil: dados demográficos. Brasília, DF: IBGE, 2010. Disponível em: http://www.ibge.gov.br. Acesso: em 12 de Jun. 2013.

LÓPEZ, C.A.A. Considerações gerais sobre plantas medicinais. Ambiente: Gestão e Desenvolvimento, v.1, n.1, p.19-27,2006. 
MARINHO, M.G.V. et al. Levantamento etnobotânico de plantas medicinais em área de caatinga no município de São José de Espinharas, Paraíba, Brasil. Revista Brasileira de Plantas Medicinais, v.13, n.2, p.170-82, 2011. Disponível em: http://www. scielo.br/scielo.php?script=sci_arttext\&pid=S1516$05722011000200008 \&$ Ing=en. Acesso em: 20 abr. 2012.

OLIVEIRA, A.K.M. et al. Ethnobotany and traditional medicine of the inhabitants of the Pantanal Negro subregion and the raizeiros of Miranda and Aquidauna, Mato Grosso do Sul, Brazil. Brazilian Journal of Biology, v.71, n.1, suppl.1:283-9, 2011.

PEREIRA, C.O. et al. Abordagem etnobotânica de plantas medicinais utilizadas em dermatologia na cidade de João Pessoa-Paraíba, Brasil. Revista Brasileira de Plantas Medicinais, v.7, n.3, p.9-17, 2005.

PESTANA, M.H.; GAGEIRO, J.N. Análise de dados para ciências sociais- a complementaridade do SPSS. $3^{a}$ Ed. Lisboa: Edições Sílabo, 2003. 670p.
PINTO, E.P.P. et al. Conhecimento popular sobre plantas medicinais em comunidades rurais de mata atlântica - Itacaré, BA, Brasil. Acta Botanica Brasilica, v.20, n.4, p.751-62, 2006.

SANTOS, R.L. et al. Análise sobre a fitoterapia como prática integrativa no Sistema Único de Saúde. Revista Brasileira de Plantas Medicinais, v.13, n.4, p.486-91,2011. Disponível em: http://www. scielo.br/scielo.php?script=sci_arttext\&pid=S151605722011000400014\&Ing=en. Acesso em: 20 abr. 2012.

TRESVENZOL, L.M. et al. Estudo sobre o comércio informal de plantas medicinais em Goiânia e cidades vizinhas. Revista Eletrônica de Farmácia, v.3, n.1, p.23-8, 2006.

VISBISKI, V.N. et al. Uso popular das plantas medicinais no assentamento Guanabara, Imbaú - PR. Publ. UEPG Ci. Exatas Terra, Ci. Agr. Eng., Ponta Grossa, v.9, n.1, p.13-20,2003. 